\title{
Locating High-Impedance Fault Section in Electric Power Systems Using Wavelet Transform, $k$-Means, Genetic Algorithms, and Support Vector Machine
}

\author{
Ying-Yi Hong and Wei-Shun Huang \\ Department of Electrical Engineering, Chung Yuan Christian University, 200 Chung Pei Road, Chung Li 320, Taiwan \\ Correspondence should be addressed to Ying-Yi Hong; yyh10632@yahoo.com.tw
}

Received 22 July 2014; Accepted 14 November 2014

Academic Editor: Vishal Bhatnaga

Copyright (C) 2015 Y.-Y. Hong and W.-S. Huang. This is an open access article distributed under the Creative Commons Attribution License, which permits unrestricted use, distribution, and reproduction in any medium, provided the original work is properly cited.

\begin{abstract}
High-impedance faults (HIFs) caused by downed conductors in electric power systems are in general difficult to be detected using traditional protection relays due to small fault currents. The energized downed conductor can pose a safety risk to the public and cause a fire hazard. This paper presents a new method for locating the line (feeder) section of the HIF with the help of limited measurements in electric power systems. The discrete wavelet transform is used to extract the features of transients caused by HIFs. A modified $k$-means algorithm associated with genetic algorithms is then utilized to determine the placement of measurement facilities. The signal energies attained by wavelet coefficients serve as inputs to the support vector machine for locating the HIF line section. The simulation results obtained from an 18-busbar distribution system show the applicability of the proposed method.
\end{abstract}

\section{Introduction}

High-impedance faults (HIFs) in general occur in electric distribution systems. HIFs occur when a conductor contacts a tree with a high-impedance or when a broken conductor touches the ground. These faults may impose fire risks and cause electric shock that endangers lives of personnel. Therefore, HIF detection is essential to ensure safety. However, detection of HIFs using traditional protection devices (e.g., overcurrent and distance relay) is difficult because the resulting level of fault current is usually smaller than the nominal current.

Lien et al. proposed a method for detecting HIFs using three-phase energy variance for the second, fourth, and sixth harmonics of unbalanced current. Then counters are designed to detect HIF arcing through statistical confidence [1]. Emanuel et al. proposed that $120 \mathrm{~Hz}$ and $180 \mathrm{~Hz}$ components may be employed to detect HIFs. The field test was supported by a simple theoretical model and laboratory measurement [2]. Kim et al. used wavelet transform to extract HIF features for developing an HIF indicator [3]. Sedighi et al. presented a statistical pattern recognition, namely, principal component analysis and Bayes classifier, for detecting HIF and discriminating it from other disturbances [4]. Lai et al. used the nearest neighbor rule approach to classify HIF and low-impedance fault (LIF) with the help of wavelet transform and voltage/current rms values [5]. Michalik et al. employed a phase displacement relation between wavelet coefficients of zero sequence voltages and currents to detect HIFs [6]. Sheng and Rovnyak used rms current, harmonic magnitudes, and phases in a decision tree for detecting HIFs [7].

On the other hand, the wavelet transform (WT) has been widely used for analyzing transient signals because of its varied window function for the time domain. The features of signals/functions can be easily extracted/decomposed via multiresolution analysis (MRA) [8]. There are many papers using discrete wavelet transform (DWT) to detect and classify PQ events [9-12]. Furthermore, artificial neural networks (ANNs) can be employed to map the input and output nonlinear relationship. The support vector machine (SVM), which is one of the ANNs, has recently been proposed for nonlinear regression and classification. Dash et al. used three SVMs for training to achieve fault classification, ground detection, and section identification, respectively, for the line 
using thyristor-controlled compensated compensators [13]. Srinivasan et al. employed SVMs with linear and polynomial kernels developed for signature extraction and device identification [14]. Janik and Lobos used space phasor for feature extraction from three-phase signals to build distinguished patterns for SVM classifiers [15]. Other applications using SVM are, for example, load forecasting [16] and transient stability analysis [17].

In previous methods, "detection" means identification of an HIF in a feeder (or transmission line) [1-4] or in one of the multiple feeders (or transmission lines) [5-7] from the secondary side of a transformer at a substation. Locating a line (feeder) section, where an HIF occurs, has not been addressed in these papers. Moreover, different features, for example, even harmonics [1], low harmonics [2,7], wavelet coefficients [3-5], voltage/current rms values [5], and phase displacement [6], were considered for detection. There was no salient result showing which features were better.

In this paper, locating the HIF line (feeder) section instead of detecting HIFs is addressed in a distribution system. Placement of multiple measurement facilities is determined first by a modified $k$-means algorithm associated with genetic algorithms. The discrete wavelet transform (DWT) is then used to extract features from these measurement locations for classification. Finally, the SVM is utilized to locate an exact HIF line section.

In Section 2, the problem description and assumptions are provided. The proposed method for locating the HIF section is given in Section 3. Simulation results obtained from an 18-busbar distribution system with HIFs are discussed in Section 4. Concluding remarks are given in Section 5.

\section{Problem Description and Assumptions}

Power engineers in general deal with the power event according to the following steps: (i) localization, (ii) classification, (iii) locating, and (iv) remedial action. These can be achieved with the help of the power supply monitoring system. When the monitored signals (voltage and current) are measured, the important features can be extracted using digital signal processing techniques. The monitoring system will assimilate the information including the features into useful knowledge/information through soft computing and machine learning for engineers to develop control strategy and to achieve decision-making.

In the last paragraph, "localization" means to identify the time for HIFs to occur. "Classification" indicates that HIFs should be discriminated from other disturbances, for example, load switching and low-impedance (short circuit) fault. "Locating" implies that an exact HIF line section should be identified. The second and third tasks will be emphasized in this paper. After locating the HIF, proper remedial actions will be activated by power engineers.

This paper deals with locating the HIF line section in a distribution system with multiple feeders using a power supply monitoring system including multiple measurement facilities at different lines. Locating a line (feeder) section, where an HIF occurs, has not been addressed in the previous papers. There are several assumptions in this paper as follows.

(i) The number of measurement facilities is given. In this paper, it is assumed that the supplier (utility) has a monitoring system including some measurement facilities that can be placed at different locations for recording.

(ii) Locating single HIF is considered. The data-window size of the signal for processing in this paper is five cycles. Simultaneous HIFs at different lines hardly occur.

(iii) Configuration of the studied distribution system is fixed. If the system topology is changed, the proposed neural network requires retraining. However, possible system configurations are generally known to engineers and the corresponding neural networks should be trained in advance.

(iv) The HIF generally occurs at a single phase of a line section. The proposed method employed MATLAB/ SIMULINK SimPowerSystems and all the three-phase transient voltages/currents at each busbar/line in the system are obtained.

\section{The Proposed Method}

The presented method includes three stages: (i) determining measurement sites, (ii) discriminating HIFs from other disturbances, and (iii) locating the HIF. The measurement sites are first determined by modified $k$-means algorithm associated with genetic algorithms. The proposed method then uses the wavelet coefficients of the currents (obtained by the measurements) as the features for classification of disturbances and the inputs of the SVM for locating the HIF.

3.1. Determination of Measurement Sites. In general, the number of power supply monitoring facilities is much smaller than the regular power, voltage, and current meters that are installed at all busbars and lines. Hence, a modified $k$-means algorithm is used to partition the system into $C$ clusters ( $C$ is the number of power supply measurement facilities). $C$ measurement facilities are placed at the lines near the pseudocenters of the $C$ clusters. This subsection describes the modified $k$-means algorithm for partitioning the system for placement of the power supply measurement facilities. Moreover, the proposed modified $k$-means algorithm is enhanced from $k$-means $[18,19]$ and fuzzy-c-means (FCM) $[20,21]$ as follows.

Let $J(U, V)$ be an objective:

$$
J(U, V)=\sum_{i=1}^{N} \sum_{c=1}^{C}\left(U_{c i}\right) \times\left\|X_{i}-V_{c}\right\|^{2},
$$

where $C$ is the number of clusters; $N$ represents the number of data (line section); $V_{c}$ signifies the vector of the center in the $c$ th clustering; $X_{i}$ is the $i$ th (known) data vector for clustering; $U_{c i}$ denotes the characteristic value ( 0 or 1$)$ as a weighting factor between $V_{c}$ and $X_{i}$. If the minimum of $J(U, V)$ is 
gained, the $N$ sets of vectors are partitioned into $C$ clusters and $V_{c}$ is formulated by

$$
V_{c}=\frac{\sum_{i=1}^{N} U_{c i} \times X_{i}}{\sum_{i=1}^{N} U_{c i}}, \quad 1 \leq c \leq C, 1 \leq i \leq N .
$$

Matrix of the characteristic values can be defined as follows:

$$
U=\left[U_{c i}\right] \in R^{C \times N} .
$$

For the $i$ th column in the matrix $U$, the sum of all elements equals one and only one element in this column is unity. The traditional $k$-means algorithm did not consider (1), which is implemented in this proposed enhanced $k$-means algorithm.

The unknown variables in the problem of placement of measurement facilities are $U_{c i}, c=1, \ldots, C$, and $i=1, \ldots, N$. Traditional optimization methods involving the gradients of objective function cannot minimize (1) because of discontinuity of the objective function. The genetic algorithm was adopted to minimize (1) herein because the genetic algorithm can deal with binary variable $U_{c i}$ efficiently [22]. The population size, crossover rate, and mutation rate in the genetic algorithm were assigned with 100, 0.9, and 0.01, respectively.

In this paper, $X_{i}$ represents one of the current vectors (signal energies calculated by DWT) caused by an HIF at a line $\ell$. The dimension $(1 \times 3660$ herein $)$ of $X_{i}$ varies with the number of studied cases. Symbols $C$ and $N$ (3660 in this paper) are the numbers of measurement facilities and the scenarios with HIFs, respectively. Let $L$ be the number of the line sections. Then $L X_{i}$ 's need to be partitioned into $C$ clusters. The vector $V_{c}(1 \times 3660)$ consisting of the virtual HIF currents serves as the center in the $c$ th cluster. All vectors of the HIF currents $X_{i}$ 's in the $c$ th cluster geometrically center at $V_{c}$. Therefore, the criterion for placing measurement facilities in the electric distribution system is as follows: place a measurement facility at line $\ell$, at which the total Euclidean distance between $X_{i}$ 's (HIFs occurring at line $\ell$ ) and $V_{c}$ is minimal, in the cth cluster.

3.2. Discrete Wavelet Transform (DWT). Fourier transform (FT) is a suitable approach for studying problems with steady state responses. Short-time Fourier transform (STFT) divides the full-time interval into a number of small/equaltime intervals, which can be individually analyzed using FT. Although the result obtained from STFT contains time and frequency information, the equal-time intervals are fixed. Thus, STFT cannot be used to detect the transient signals. On the other hand, the discrete wavelet transform (DWT) has been widely used for analyzing the transient signals due to its varied scale and wavelet functions [23-25]. The features of signals can be easily extracted via the multiresolution analysis (MRA). DWT avoids the disadvantages of both FT and STFT.

A signal can be represented as a sum of wavelet functions $\varphi(t)$ and scale functions $\phi(t)$ with coefficients at different time shifts and scales (frequencies) using DWT. DWT can extract the features of transient signals by decomposing signal components overlapping in both time and frequency [8].
According to DWT, a time-varying function (signal) $f(t) \epsilon$ $L^{2}(R)$ can be expressed as follows:

$$
\begin{aligned}
f(t)= & \sum_{k} c_{0}(k) \phi(t-k)+\sum_{k} \sum_{j=1} d_{j}(k) 2^{-j / 2} \varphi\left(2^{-j} t-k\right) \\
= & \sum_{k} c_{j_{0}}(k) 2^{-j_{0} / 2} \phi\left(2^{-j_{0}} t-k\right) \\
& +\sum_{k} \sum_{j=j_{0}} d_{j}(k) 2^{-j / 2} \varphi\left(2^{-j} t-k\right),
\end{aligned}
$$

where $c_{0}$ and $d_{j}$ represent the scaling (coarse) coefficient at scale 0 and wavelet (detailed) coefficient at scale $j$, respectively. The symbol $k$ represents the translation coefficient. The scales $j=1,2, \ldots$ denote the different (high to low) frequency bands. The variable $j_{o}$ is an integer. The translated and scaled (dilated) version of the wavelet, $\varphi\left(2^{-j} t-k\right)$, used in the multiresolution analysis (MRA), constructs a time-frequency picture of the signal.

There are some other wavelets in the wavelet theory [8]: Haar wavelets have compact support (a finite bounded set) but are discontinuous. Shannon wavelets are very smooth but are not compactly supported and they decay at infinity very slowly. Compared with these wavelets, Daubechies- 4 belongs to a class of orthonormal basis-generating, continuous, and compactly supported wavelets. Daubechies- 4 is adopted in this paper to extract the features of the line currents at scales 1,2 , and 3 with a sampling rate of 128 points/cycle.

3.3. Multiresolution Analysis (MRA). As shown in (4), $f(t)$ is constructed by $\phi(t)$ and decomposed by $\varphi(t)$ at different scales (resolution levels). $\varphi(t)$ generates the detailed version of $f(t)$ and $\phi(t)$ generates the coarse version of $f(t)$. It can be shown that $[8]$

$$
\begin{aligned}
c_{j+1}(k) & =\sum_{m} h(m-2 k) c_{j}(m), \\
d_{j+1}(k) & =\sum_{m} h_{1}(m-2 k) c_{j}(m),
\end{aligned}
$$

where $h(m-2 k)$ and $h_{1}(m-2 k)$ are the low-pass and highpass filters, respectively [8]. These two equations show that the scaling and wavelet coefficients at different scale levels can be obtained by convolving the expansion coefficients at scale $j$ by the time-reversed recursion coefficients $h(\cdot)$ and $h_{1}(\cdot)$ and then downsampling or decimating to give the expansion coefficients at the next level of $j+1$. The term "downsampling" indicates that the number at lower scale $j$ is double compared with that at higher scale $j+1$ due to the filters $h(m-2 k)$ and $h_{1}(m-2 k)$. This process is called the "analysis (decomposition)" from the fine scale to the coarse scale. The reverse process, called synthesis (construction), from the coarse scale to the fine scale, is omitted here. Figure 1 illustrates a three-scale MRA decomposition for a signal. The symbols $h, h_{1}$, and " $\downarrow 2$ " denote the low-pass filter, high-pass filter, and "downsampling," respectively.

The small scales represent high-frequency ranges. Only the wavelet coefficient $\left(d_{j}\right)$ is regarded as a feature due to 


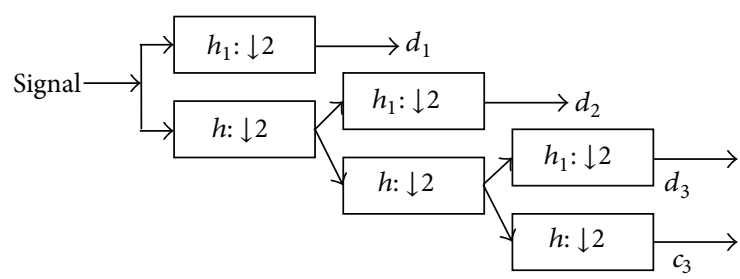

Figure 1: A three-scale MRA decomposition for a signal.

the high-frequency phenomena from HIFs. More specifically, if the sampling rate from the measurement facility is 128 points/cycle, then scales 1,2 , and 3 cover $3.84 \sim 1.92 \mathrm{kHz}, 1.92 \sim$ $0.96 \mathrm{kHz}$, and $0.96 \sim 0.48 \mathrm{kHz}$, respectively. Lower harmonics were not considered for the SVM because they (with large values) do not provide significant discrimination among lines.

3.4. Parseval Theorem. When the MRA is applied to a transient signal, a large amount of wavelet coefficients will be attained. Although the wavelet coefficients are useful, it is difficult for SVM to train/validate that much information. More specifically, if sampling rate is 128 points/cycle and five cycles are utilized, the numbers of wavelet coefficients at scales 1, 2, and 3 are 320, 160, and 80 due to "downsampling," respectively. Implementing $560 \times C$ input neurons in an SVM becomes impractical, where $C$ is the number of measurement facilities defined in Section 3.1. A trade-off treatment using Parseval's theorem is presented in this paper:

$$
\int|f(t)|^{2} d t=\sum_{k}\left|c_{j_{0}}(k)\right|^{2}+\sum_{k} \sum_{j=1}\left|d_{j}(k)\right|^{2}
$$

In this paper, only $\sum_{k} \sum_{j=1}\left|d_{j}(k)\right|^{2}$ in (7) is calculated because the HIF belongs to transients. This term is called "current energy" or simply "energy" in this paper. Applications of $\sum_{k} \sum_{j=1}\left|d_{j}(k)\right|^{2}$ are as follows.

(i) Determination of measurement facility placement: $\sum_{k} \sum_{j=1}\left|d_{j}(k)\right|^{2}$ is computed for each line section to be an element of $X_{i}$ for a given scenario described in Section 3.1. The number of given scenarios is 3660 which will be discussed in Section 4.1.

(ii) Feature extraction of transient signals: $\sum_{k} \sum_{j=1}\left|d_{j}(k)\right|^{2}$ is separated into the first to third scales $\left(d_{1} \sim d_{3}, j=\right.$ 1,2, and 3) for an HIF current at each line section. These features will serve as inputs for SVM.

3.5. Support Vector Machine (SVM). Traditional multilayer neural networks have some limitations: (i) many inputs due to need of diversity for inputs, (ii) requirement of crucial features for inputs, (iii) trial and error for number of neurons in the hidden layer, and (iv) multimodal with many local minimums. Avoiding the above demerits, SVM is a supervised artificial neural network designed for solving classification problems $[26,27]$. In essence, SVM maximizes the margin between the training data and the decision boundary, which can be formulated as a quadratic optimization problem. The subset of patterns that are closest to the decision boundary is called the support vector.

SVM maximizes the separating margin between two classes, given by a set of $P$ data pairs $\left(x_{p}, c_{p}\right)$, where $x_{p}$ and $c_{p}$ denote the input vector and class, $p=1,2, \ldots, P$, respectively. For linear separable training pairs of two classes, the separating hyperplane $h(x)$ is given by

$$
h(x)=w^{t} x+b=0,
$$

where $w$ and $b$ are the vectors of weighting factors and biases, respectively. If a nonlinear hyperplane $\psi(\cdot)$ is considered, then

$$
h(x)=w^{t} \psi(x)+b=0 .
$$

The maximal separating margin can be attained by minimizing the following primal problem if two classes are not linearly distributed [28]:

$$
\begin{array}{ll}
\min & \frac{1}{2} w^{t} w+K \sum_{p=1}^{P} \xi_{p} \\
\text { subject to } & c_{p}\left(w^{t} \psi\left(x_{p}\right)+b\right) \geq 1-\xi_{p}, \quad p=1,2, \ldots, P,
\end{array}
$$

where $\xi_{p}$ is the so-called fulfilling variable. The symbol $K$ is a regularization parameter. In order to search a proper $K$, performance of the trained SVM needs assessment as follows. The training data are divided into two sets. One is used to train the SVM while the other, called the validation set, is used for evaluating the SVM. According to the performance on the validation set, a proper value of $K$ can be attained.

Equations (10) and (11) can be transformed into the unconstrained Lagrangian:

$$
\begin{aligned}
L(w, b, \xi, \mu)= & \frac{1}{2} w^{t} w+K \sum_{p=1}^{P} \xi_{p} \\
& +\sum_{p=1}^{P} \mu_{p}\left[c_{p}\left(w^{t} \psi\left(x_{p}\right)+b\right)-1+\xi_{p}\right],
\end{aligned}
$$

where $\mu_{p}$ is the dual variable (Lagrange multiplier) for inequality constraint, (11). Obviously, the form of (11) is the same as the output of a neuron if $\varphi(\cdot)$ and $\xi_{p}$ are considered the activating function of a neuron and nonnegative slack variable, respectively.

\section{Simulation Results}

4.1. Simulation Data. The applicability of the proposed methodology is verified by simulation results in this section. An 18-busbar radial system with 17 line sections illustrated in Figure 2 serves as a sample system in this paper. Its busbar and line data are provided in [29]. To train the SVM, the original load level was varied within $\pm 10 \%$ (61 conditions) and the HIFs occur at different angles within $0^{\circ} \sim 359^{\circ}$ ( 4 conditions) 


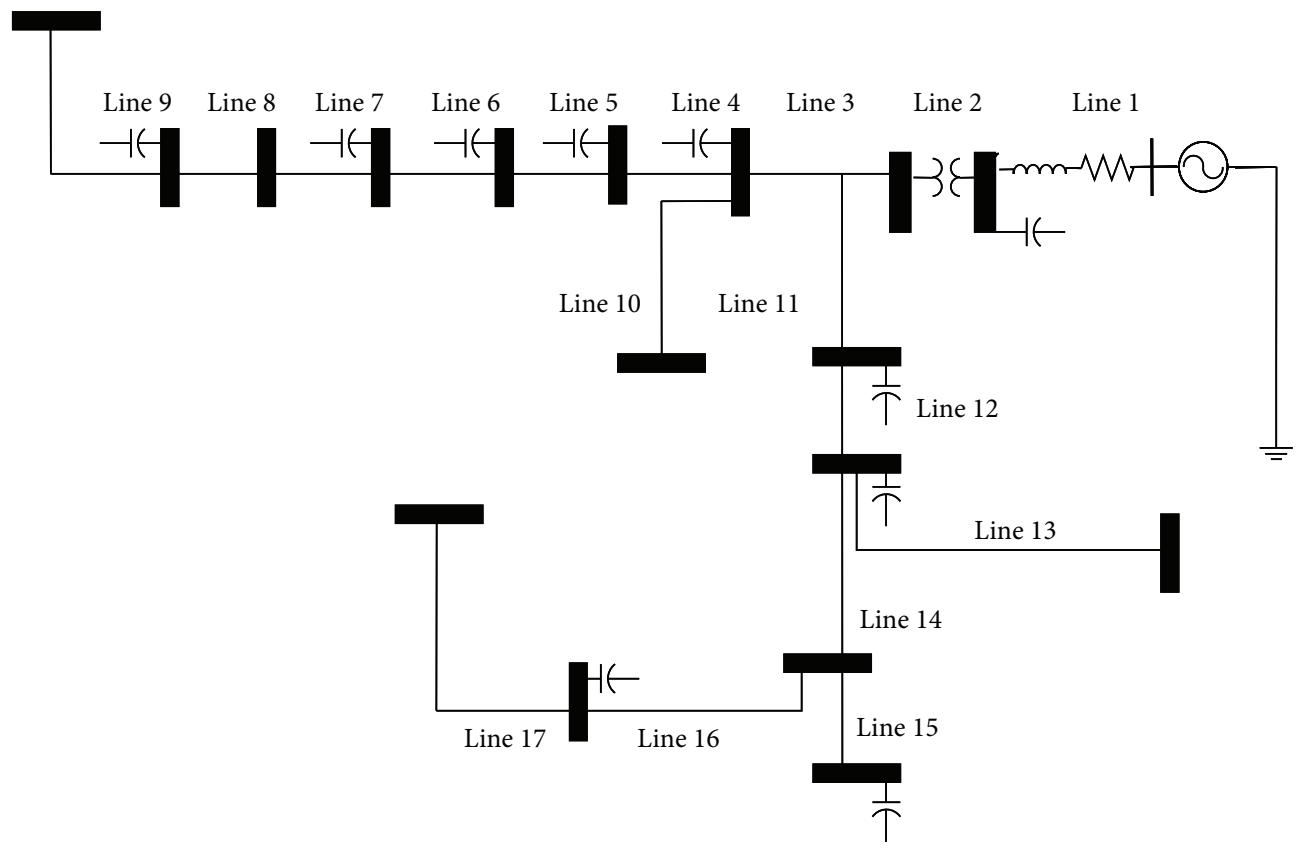

Figure 2: One-line diagram for studied distribution system.

and at 15 different line sections for obtaining a total of 3660 $(=61 \times 4 \times 15)$ data. $70 \%, 10 \%$, and $20 \%$ of these 3660 data were used stochastically for training, validating, and testing the proposed SVM, respectively. The arc of HIF was modeled with two antiparallel DC sources and diodes which were connected to a random resistor [2]. The proposed methods were implemented by MATLAB 7.0 (SimPowerSystems) on a C2D (Core 2 Due) $2.13 \mathrm{GHz}$ computer (RAM 3.5 G). The data-window size of the signal for processing in this paper is five cycles.

Because the power supply measurement facilities are more expensive than general meters, the number of measurement facilities is limited. Discussion of purchasing the measurement facilities and determination of a proper number for the measurement facilities are beyond the scope of this paper. Hence, 14, 11, 8, 4, and 2 measurement facilities (i.e., C) are assumed to be available in this paper. Table 1 illustrates the SVM information associated with measurements. Because HIF energies of the first to third scales $\left(d_{1} \sim d_{3}\right)$ were considered, the number of input neurons equals measurements $(C)$ multiplied by 3 . These are cases 1 5. Moreover, the current at the neural line of the main transformer is generally available and can be utilized. These are cases $6 \sim 10$. Finally, four binary bits are sufficient for discriminating 15 line sections excluding primary sides (line 1) and the main transformer (denoted by line 2) in this system.

4.2. Feature Extraction by DWT. As described in Sections 3.3 and 3.4, the "energies" for HIF currents of the first to third scales at each line section are used as features for SVM inputs. Assume that an HIF occurs $\left(90^{\circ}\right)$ at line section 12 . Figure 3 shows the energy distribution of the neighborhood of line section 12 (i.e., line sections 11 and 13). The energies for the
TABLE 1: SVM information associated with measurements.

\begin{tabular}{lccc}
\hline & Facility number $(C)$ & Input neurons & Output neurons \\
\hline Case 1 & 2 & $2 \times 3$ & 4 \\
Case 2 & 4 & $4 \times 3$ & 4 \\
Case 3 & 8 & $8 \times 3$ & 4 \\
Case 4 & 11 & $11 \times 3$ & 4 \\
Case 5 & 14 & $14 \times 3$ & 4 \\
Case 6 & $2+1$ & $3 \times 3$ & 4 \\
Case 7 & $4+1$ & $5 \times 3$ & 4 \\
Case 8 & $8+1$ & $9 \times 3$ & 4 \\
Case 9 & $11+1$ & $12 \times 3$ & 4 \\
Case 10 & $14+1$ & $15 \times 3$ & 4 \\
\hline
\end{tabular}

first to sixth scales at these three line sections are shown. It is apparent that the normal energy and HIF energy are almost the same for the current of the fourth (also for fifth and sixth) scale. Hence, current energies for the fourth to sixth scales cannot serve as features and only current energies for the first to third scales are considered further. Please note that the energies are normalized to be per unit and are in terms of $\log _{10}$ because the energies of the fifth and sixth scales are much larger than those of other scales.

4.3. Scaled Energies at Line Sections. An HIF occurring at line section 12 is discussed in this section. Figure 4 illustrates the HIF currents in terms of the DWT-scaled energy distributions at each line section. The vertical axis denotes the energy magnitude while the horizontal axis means the $d_{1} \sim d_{3}$ at each line section. Please note that the energies are normalized to be per unit. 


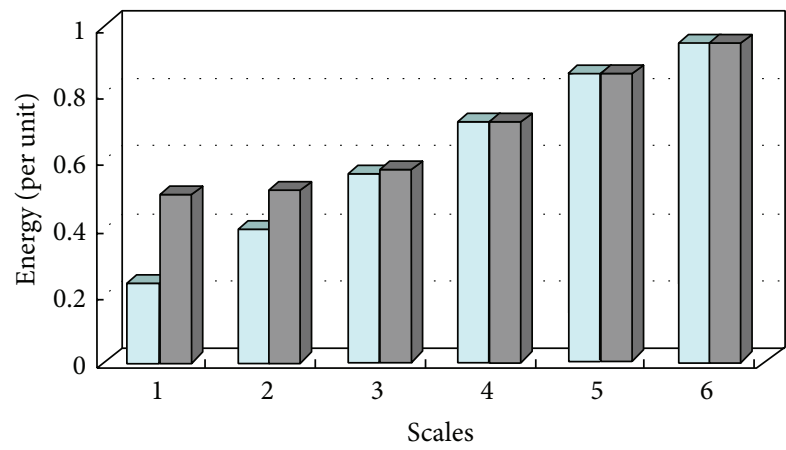

口 Normal - HIF

(a) 1st-6th normal and HIF energies at line section 11

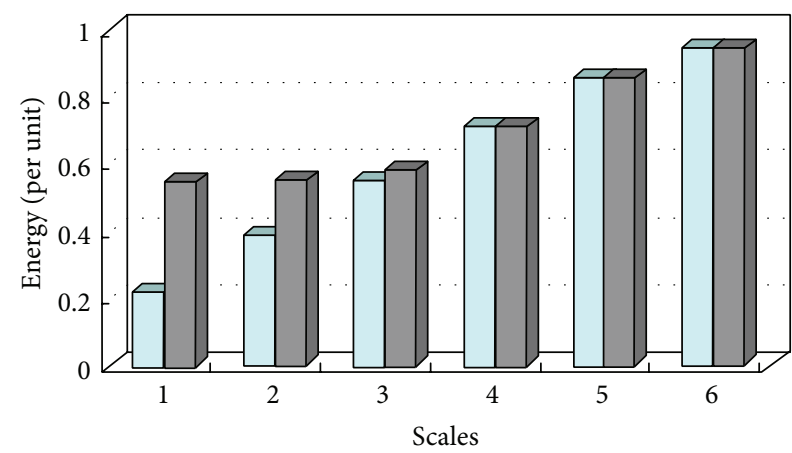

口 Normal

- HIF

(b) 1st-6th normal and HIF energies at line section 12

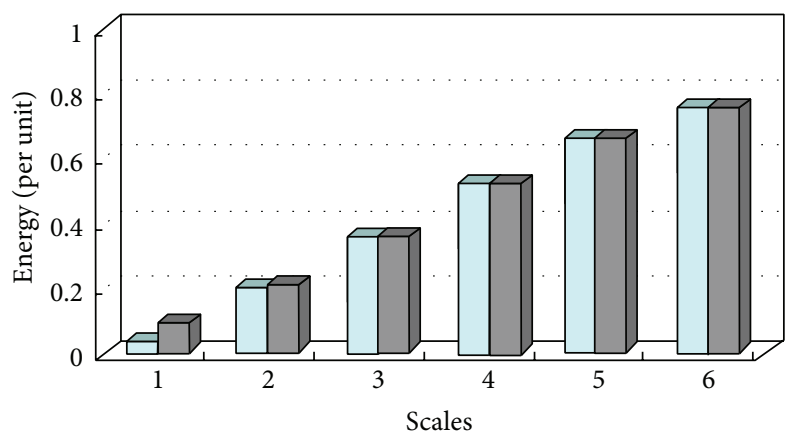

Normal

口 HIF

(c) 1st-6th normal and HIF energies at line section 13

FIGURE 3: Energy distributions near faulted line.
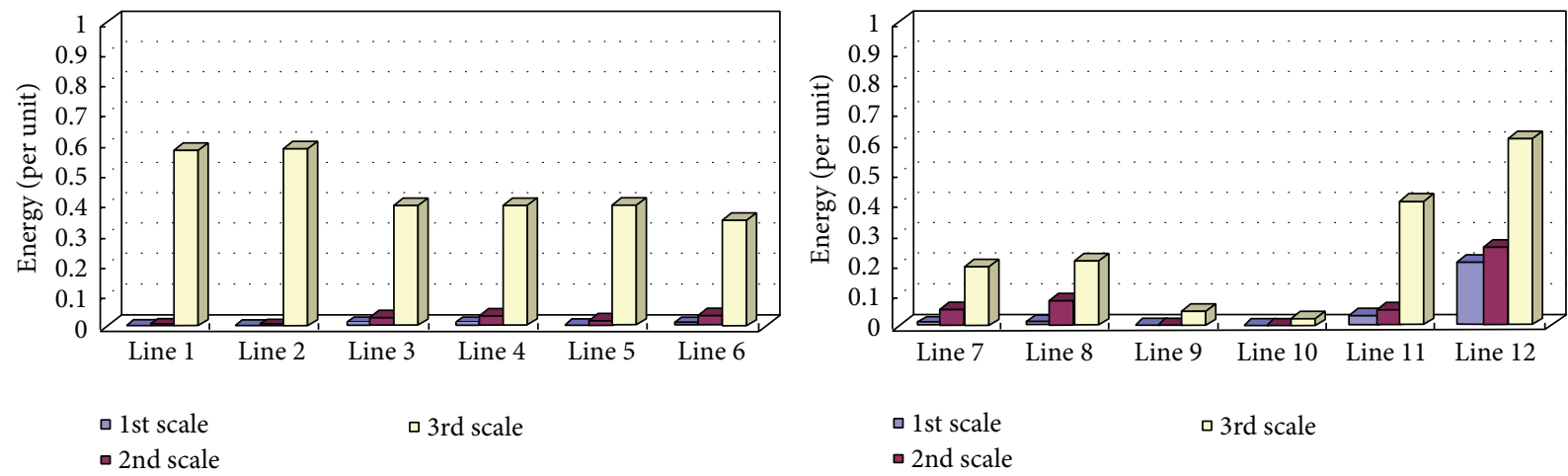

- 2nd scale

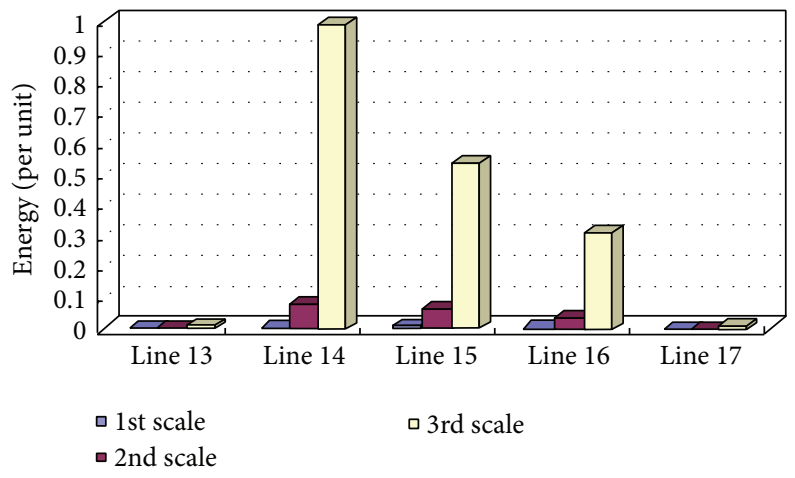

FIgURE 4: HIF energy distributions at all line sections. 
TABLE 2: Different $C s$ and corresponding clusters ( $k$-means).

\begin{tabular}{cc}
\hline$C$ & Line clusters \\
\hline 14 & $(1,2),(3),(4),(5),(6),(7),(8),(9),(10),(11,12),(13),(14)$, \\
& $(15,16),(17)$ \\
11 & $(1,2),(3),(4,5,6),(7,8),(9),(10),(11,12),(13),(14),(15$, \\
& $16),(17)$ \\
8 & $(1,2),(3),(4,5,6),(7,8),(9),(10),(11,12),(13,14,15,16,17)$ \\
4 & $(1,2),(3,4,5,6,7,8,9,11,12),(10),(13,14,15,16,17)$ \\
2 & $(1,2),(3,4,5,6,7,8,9,10,11,12,13,14,15,16,17)$ \\
\hline
\end{tabular}

TABLE 3: Different Cs and corresponding clusters (FCM).

\begin{tabular}{cc}
\hline$C$ & Line clusters \\
\hline 14 & $\begin{array}{c}(1),(2),(3),(4),(5),(6),(7),(8),(9,10,13,17),(11),(12), \\
(14),(16)\end{array}$ \\
11 & $\begin{array}{c}(1,2),(3),(4),(5),(6),(7,8),(9,10,13,17),(11),(12),(14), \\
(15,16)\end{array}$ \\
8 & $(1,2),(3),(4,5,6),(7,8),(9,10,13,17),(11),(12),(14,15,16)$ \\
4 & $(1,2),(3,4,5,6),(7,8,11,12),(9,10,13,14,15,16,17)$ \\
2 & $(1,2,3,4,5,6),(7,8,9,10,11,12,13,14,15,16,17)$ \\
\hline
\end{tabular}

When the busbar load varies and the HIF occurs at distinct angles, the above phenomena will be discriminated. Hence, the "energies" of the HIF currents of the first to third scales are important features for locating the HIF line section.

4.4. Measurement Facility Placement. Because 14, 11, 8, 4, and 2 power supply measurement facilities are assumed to be available in the test sample, $C$ may be $14,11,8,4$, or 2 and $N=3660$, respectively ( $C$ and $N$ were defined in Section 3.1). For a given $C$, the same 3660 sets of data were employed to perform the HIF current energy clustering by the modified $k$-means algorithm. Table 2 illustrates the different $C$ 's and corresponding clusters. Each cluster is quoted by parentheses. The line section with an italic font in Table 2 denotes the one installed with a measurement facility. Traditional FCM is also employed to study the line section clustering as shown in Table 3. As can be seen, some clusters obtained by the FCM are infeasible because lines in a cluster may not be adjacent to each other. For example, in the last row of Table 3, line sections 7, 8, and 9 in a cluster are not adjacent to line sections 10 17.

More specifically, the condition for $C=2$ and $c=2$ in the last row of Table 2 is described here: Table 4 illustrates the distances (norm) between $X_{i}, i=3,4, \ldots, 17$, and its clustering center $V_{2}(c=2)$ for $C=2$. As can be seen, the distance between $X_{12}$ and $V_{2}$ is the smallest. Therefore, the measurement facility is placed at line section 12 .

In this paper, the dimension of $X_{i}$ is $1 \times 3660$ where 3660 is the number of scenarios from the test system. Each element of $X_{i}$ is the total energy at line section $i$ for one of the 3660 cases. Moreover, there are $17 X_{i}$ 's (number of lines) for the 18 -busbar system where 17 is the line number.

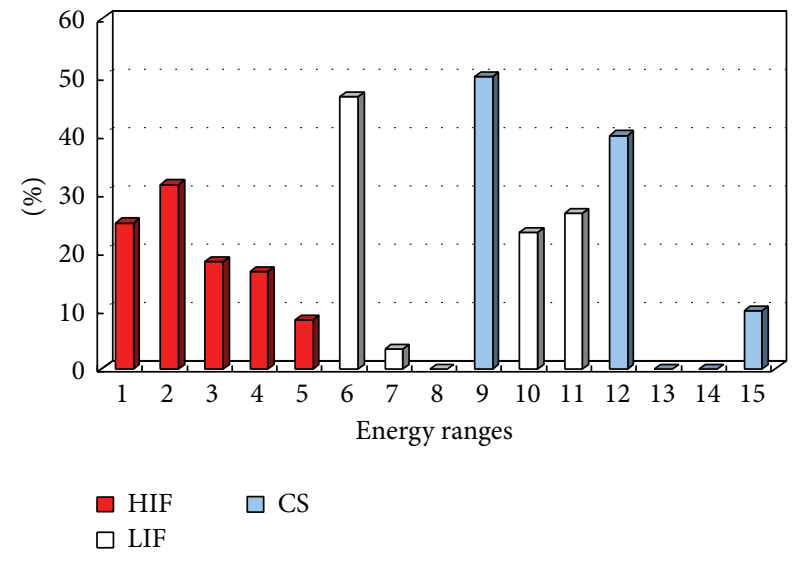

FIGURE 5: Total energy disturbances for 3 different disturbances.

4.5. Classification among HIF, Short Circuit, and Switching. There are 930 cases with short circuits (low-impedance faults, LIFs) and 620 cases with capacitor switching (CS) for further investigation of energy distributions. Figure 5 shows the total energy of the first to third scales for different HIFs, LIFs, and CS. The vertical axis denotes the percentage of occurrence for the three individual disturbances. The horizontal axis includes 15 energy ranges $\left(\log _{10}\right)$. More specifically, ranges 1 , 2 , and 15 represent $3.48-3.56,3.57-3.65$, and $10.09-10.79$ p.u., respectively. It can be found that HIFs include smaller total energies from range 1 to range 5 . Hence, the total energy can be employed to discriminate HIFs from other disturbances, for example, LIF and CS.

4.6. Accuracy of Locating HIFs by SVM. As described in Section $4.1,70 \%, 10 \%$, and $20 \%$ of the 3660 data are used stochastically for training, validating, and testing, respectively. Table 5 illustrates the number of iterations, CPU time for training the SVM, and the accuracy rate for the 10 cases defined in Table 1 . The following comments can be drawn from Table 5.

(i) The numbers of iterations for all cases are almost the same for the SVM despite the different number of input neurons.

(ii) The CPU time required varies with the number of input neurons.

(iii) Accuracy rates are greater than $99 \%$ except for cases 1 and 6.

(iv) Cases 1 and 6 with only two measurement facilities still gain accuracy rate of $97.4 \%$ and $98.36 \%$, respectively. This result ensures the advantage of the proposed method using the SVM.

(v) The neutral current at the substation improves accuracy rates only for cases 1 and 2 (corresponding to cases 6 and 7) with fewer measurements. 
TABLE 4: Distances $\left(\times 10^{5}\right)$ between $X_{i}$ and its clustering center.

\begin{tabular}{|c|c|c|c|c|c|c|c|c|c|c|c|c|c|c|}
\hline$X_{3}$ & $X_{4}$ & $X_{5}$ & $X_{6}$ & $X_{7}$ & $X_{8}$ & $X_{9}$ & $X_{10}$ & $X_{11}$ & $X_{12}$ & $X_{13}$ & $X_{14}$ & $X_{15}$ & $X_{16}$ & $X_{17}$ \\
\hline 746.02 & 577.24 & 699.47 & 666.77 & 316.63 & 292.76 & 564.36 & 575.65 & 202.95 & 189.19 & 578.97 & 342.92 & 548.87 & 547.7 & 578.97 \\
\hline
\end{tabular}

TABLE 5: Performance of SVM.

\begin{tabular}{lccc}
\hline & Iterations number & CPU sec. & Accuracy rate (\%) \\
\hline Case 1 & 32 & 170 & 97.40 \\
Case 2 & 29 & 163 & 99.18 \\
Case 3 & 33 & 226 & 99.86 \\
Case 4 & 30 & 234 & 99.72 \\
Case 5 & 30 & 260 & 99.72 \\
Case 6 & 32 & 171 & 98.36 \\
Case 7 & 29 & 171 & 99.72 \\
Case 8 & 33 & 234 & 99.86 \\
Case 9 & 30 & 241 & 99.72 \\
Case 10 & 31 & 279 & 99.72 \\
\hline
\end{tabular}

\section{Conclusions}

This paper proposed a new method for locating the line section with an HIF using DWT, modified $k$-means, and SVM. Compared with the existing methods involving identification of an HIF in a feeder (or transmission line) or in one of the multiple feeders from the secondary side of a substation, the proposed approach is able to locate an HIF line section in a distribution system with multiple feeders using a power supply monitoring system including multiple power supply measurement facilities at different lines. Classification of disturbances and locating the HIF are addressed.

The features (current energies) at three distinct scales (frequency bands) were extracted by MRA in DWT. These features provide important information for the SVM to locate the line section with an HIF. Moreover, the energies of HIF are discriminated obviously from those of LIF and CS.

The simulation results obtained from an 18-busbar distribution system show that good accuracy can still be attained using only a few measurements (e.g., two in this paper) due to the SVM. Hence, SVM is very useful when only a few measurements are available.

\section{Conflict of Interests}

The authors declare that there is no conflict of interests regarding the publication of this paper.

\section{Acknowledgment}

The authors gratefully acknowledge the financial support from the National Science Council, Taiwan, under Grant no. 96-2221-E-033-069-MY2.

\section{References}

[1] K. Y. Lien, S. L. Chen, C. J. Liao, T. Y. Guo, T. M. Lin, and J. S. Shen, "Energy variance criterion and threshold tuning scheme for high impedance fault detection," IEEE Transactions on Power Delivery, vol. 14, no. 3, pp. 810-817, 1999.
[2] A. E. Emanuel, D. Cyganski, J. A. Orr, S. Shiller, and E. M. Gulachenski, "High impedance fault arcing on sandy soil in $15 \mathrm{kV}$ distribution feeders: contributions to the evaluation of the low frequency spectrum," IEEE Transactions on Power Delivery, vol. 5, no. 2, pp. 676-686, 1990.

[3] C. H. Kim, H. Kim, Y. H. Ko, S. H. Byun, R. K. Aggarwal, and A. T. Johns, "A novel fault-detection technique of high-impedance arcing faults in transmission lines using the wavelet transform," IEEE Transactions on Power Delivery, vol. 17, no. 4, pp. 921-929, 2002.

[4] A.-R. Sedighi, M.-R. Haghifam, O. P. Malik, and M.-H. Ghassemian, "High impedance fault detection based on wavelet transform and statistical pattern recognition," IEEE Transactions on Power Delivery, vol. 20, no. 4, pp. 2414-2421, 2005.

[5] T. M. Lai, L. A. Snider, E. Lo, and D. Sutanto, "High-impedance fault detection using discrete wavelet transform and frequency range and RMS conversion," IEEE Transactions on Power Delivery, vol. 20, no. 1, pp. 397-407, 2005.

[6] M. Michalik, W. Rebizant, M. R. Lukowicz, S. J. Lee, and S. H. Kang, "High-impedance fault detection in distribution networks with use of wavelet-based algorithm," IEEE Transactions on Power Delivery, vol. 21, no. 4, pp. 1793-1802, 2006.

[7] Y. Sheng and S. M. Rovnyak, "Decision tree-based methodology for high impedance fault detection," IEEE Transactions on Power Delivery, vol. 19, no. 2, pp. 533-536, 2004.

[8] C. S. Burrus, R. A. Gopinath, and H. Guo, Introduction to Wavelets and Wavelet Transforms, Prentice Hall, Upper Saddle River, NJ, USA, 1998.

[9] A. Elmitwally, S. Farghal, S. Abdelkader, and M. Elkateb, "Proposed wavelet-neurofuzzy combined system for power quality violations detection and diagnosis," IEE Proceedings: Generation, Transmission and Distribution, vol. 148, no. 1, pp. 15-20, 2001.

[10] L. Angrisani, P. Daponte, M. D’Apuzzo, and A. Testa, "A measurement method based on the wavelet transform for power quality analysis," IEEE Transactions on Power Delivery, vol. 13, no. 4, pp. 990-998, 1998.

[11] S. Santoso, E. J. Powers, W. M. Grady, and A. C. Parsons, "Power quality disturbance waveform recognition using wavelet-based neural classifier. Part 1. theoretical foundation," IEEE Transactions on Power Delivery, vol. 15, no. 1, pp. 222-228, 2000.

[12] H. Mokhtari, M. Karimi-Ghartemani, and M. R. Iravani, "Experimental performance evaluation of a wavelet-based online voltage detection method for power quality applications," IEEE Transactions on Power Delivery, vol. 17, no. 1, pp. 161-172, 2002.

[13] P. K. Dash, S. R. Samantaray, and G. Panda, "Fault classification and section identification of an advanced series-compensated transmission line using support vector machine," IEEE Transactions on Power Delivery, vol. 22, no. 1, pp. 67-73, 2007.

[14] D. Srinivasan, W. S. Ng, and A. C. Liew, "Neural-network-based signature recognition for harmonic source identification," IEEE Transactions on Power Delivery, vol. 21, no. 1, pp. 398-405, 2006. 
[15] P. Janik and T. Lobos, "Automated classification of powerquality disturbances using SVM and RBF networks," IEEE Transactions on Power Delivery, vol. 21, no. 3, pp. 1663-1669, 2006.

[16] S. Fan and L. Chen, "Short-term load forecasting based on an adaptive hybrid method," IEEE Transactions on Power Systems, vol. 21, no. 1, pp. 392-401, 2006.

[17] L. S. Moulin, A. P. Alves Da Silva, M. A. El-Sharkawi, and R. J. Marks II, "Support vector machines for transient stability analysis of large-scale power systems," IEEE Transactions on Power Systems, vol. 19, no. 2, pp. 818-825, 2004.

[18] N. K. Bose and P. Liang, Neural Network Fundamentals with Graphs, Algorithms, and Applications, McGraw-Hill, New York, NY, USA, 1996.

[19] Y. K. Lam and P. W. M. Tsang, "eXploratory K-Means: a new simple and efficient algorithm for gene clustering," Applied Soft Computing Journal, vol. 12, no. 3, pp. 1149-1157, 2012.

[20] T. Velmurugan, "Performance based analysis between k-Means and Fuzzy C-Means clustering algorithms for connection oriented telecommunication data," Applied Soft Computing Journal, vol. 19, pp. 134-146, 2014.

[21] J. C. Bezdek, R. Ehrlich, and W. Full, "FCM: the fuzzy $c$-means clustering algorithm," Computers \& Geosciences, vol. 10, no. 2-3, pp. 191-203, 1984.

[22] D. Datta, "Unit commitment problem with ramp rate constraint using a binary-real-coded genetic algorithm," Applied Soft Computing Journal, vol. 13, no. 9, pp. 3873-3883, 2013.

[23] M. Jamil, A. Kalam, A. Q. Ansari, and M. Rizwan, "Generalized neural network and wavelet transform based approach for fault location estimation of a transmission line," Applied Soft Computing, vol. 19, pp. 322-332, 2014.

[24] D. Bayram and S. Şeker, "Wavelet based Neuro-Detector for low frequencies of vibration signals in electric motors," Applied Soft Computing Journal, vol. 13, no. 5, pp. 2683-2691, 2013.

[25] H. Eristi, "Fault diagnosis system for series compensated transmission line based on wavelet transform and adaptive neurofuzzy inference system," Measurement, vol. 46, no. 1, pp. 393401, 2013.

[26] S. Ekici, "Support Vector Machines for classification and locating faults on transmission lines," Applied Soft Computing Journal, vol. 12, no. 6, pp. 1650-1658, 2012.

[27] M. Matsumoto and J. Hori, "Classification of silent speech using support vector machine and relevance vector machine," Applied Soft Computing Journal, vol. 20, pp. 95-102, 2014.

[28] K. S. Chua, "Efficient computations for large least square support vector machine classifiers," Pattern Recognition Letters, vol. 24, no. 1-3, pp. 75-80, 2003.

[29] W. M. Grady, M. J. Samotyj, and A. H. Noyola, "Minimizing network harmonic voltage distortion with an active power line conditioner," IEEE Transactions on Power Delivery, vol. 6, no. 4, pp. 1690-1697, 1991. 


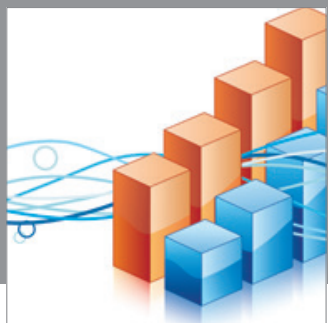

Advances in

Operations Research

mansans

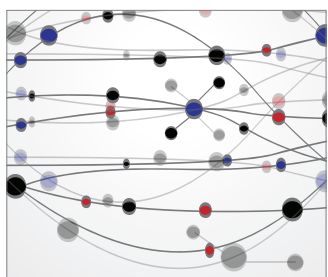

The Scientific World Journal
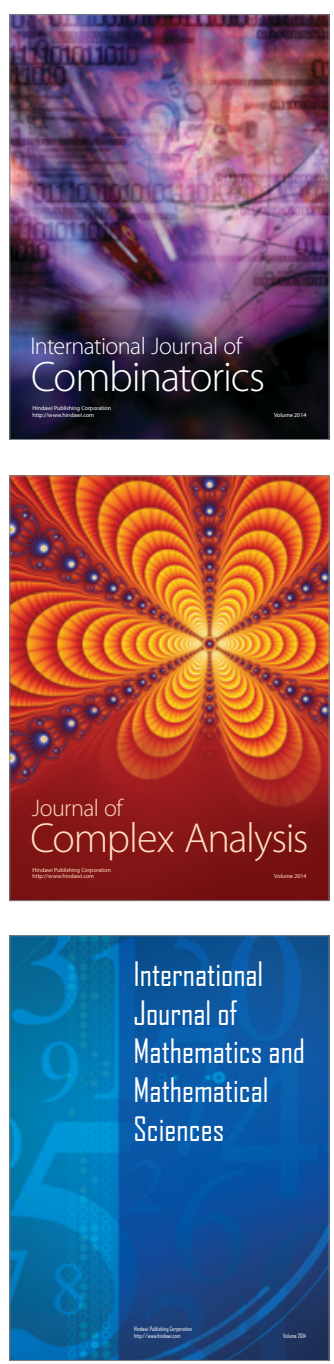
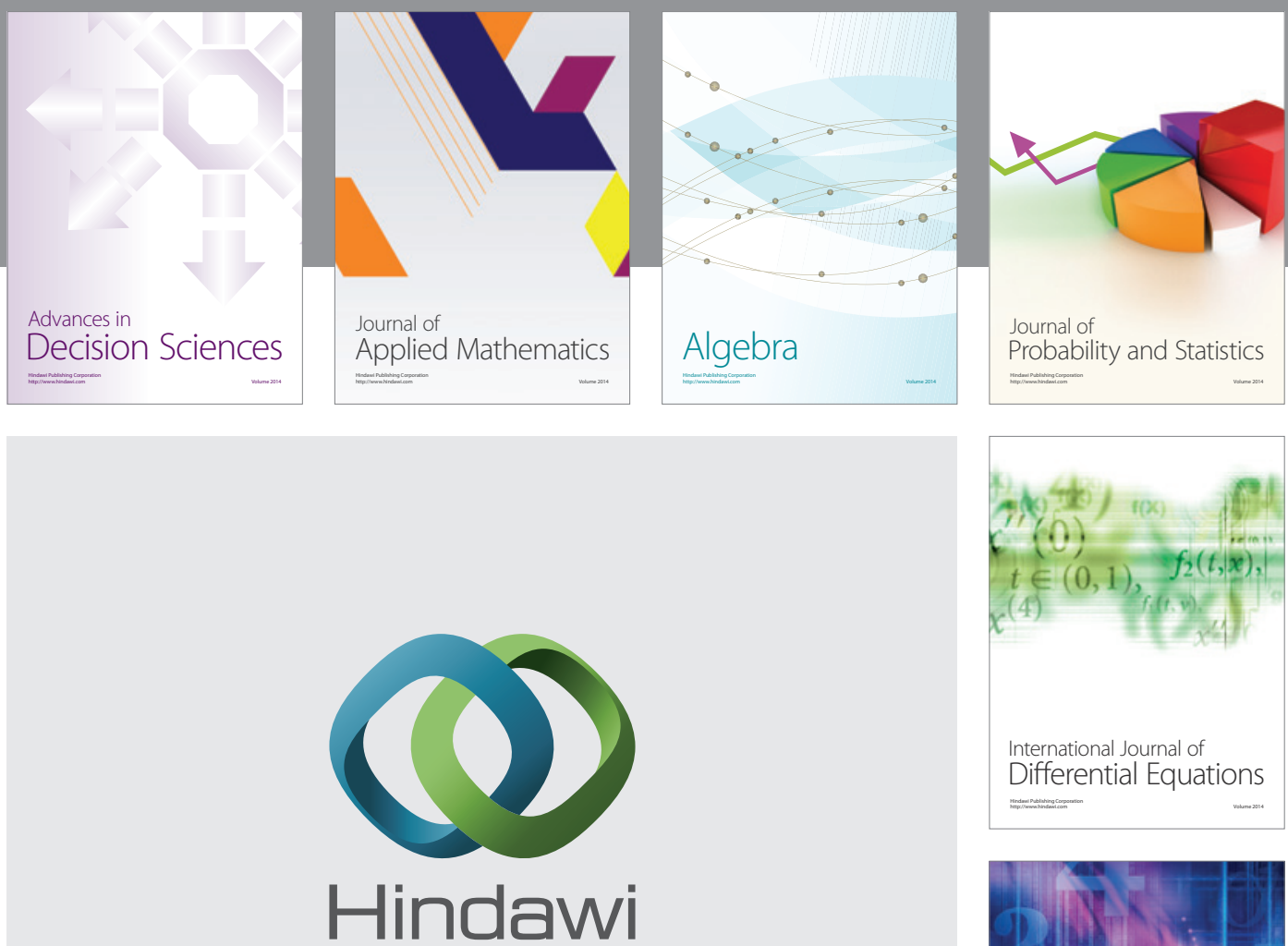

Submit your manuscripts at http://www.hindawi.com
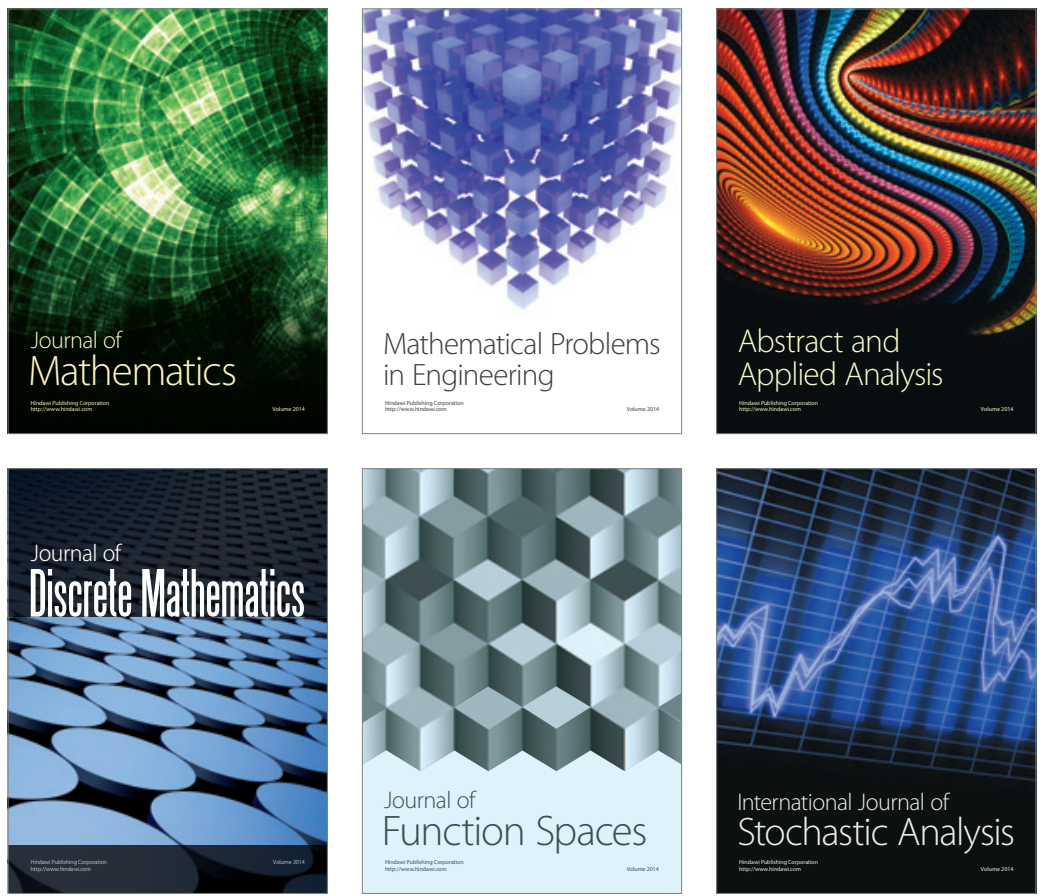

Journal of

Function Spaces

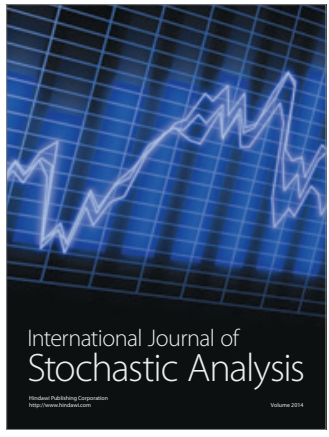

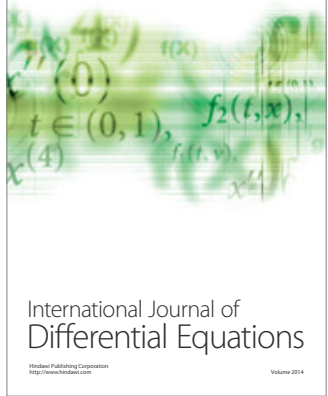
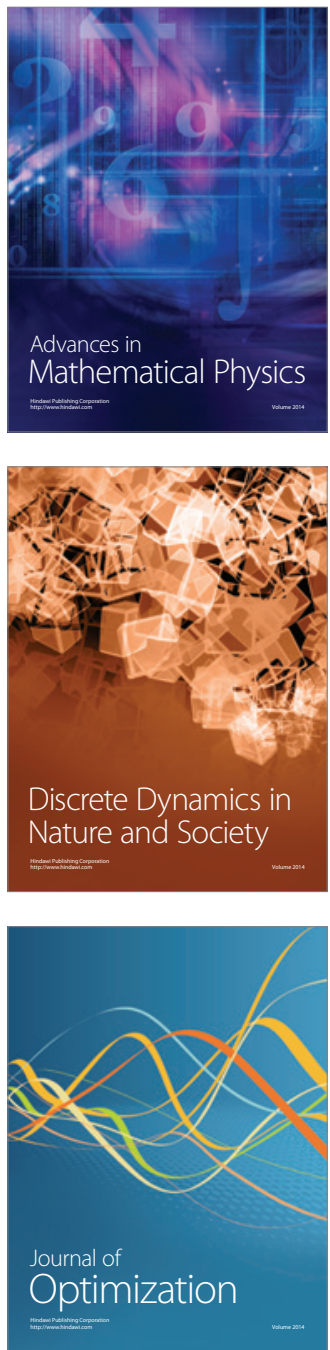